\title{
ssc-miR-185 targets cell division cycle 42 and promotes the proliferation of intestinal porcine epithelial cell
}

\author{
Wei Wang ${ }^{1}$, Pengfei Wang ${ }^{1}$, Kaihui Xie ${ }^{1}$, Ruirui Luo ${ }^{1}$, Xiaoli Gao ${ }^{1}$, Zunqiang Yan ${ }^{1}$, \\ Xiaoyu Huang ${ }^{1}$, Qiaoli Yang ${ }^{1}$, and Shuangbao Gun ${ }^{1,2, *}$
}

\footnotetext{
* Corresponding Author: Shuangbao Gun Tel: +86-0931-763-1804,

Fax: +86-0931-763-2459

E-mail: gunsbao056@126.com
}

${ }^{1}$ College of Animal Science and Technology, Gansu Agricultural University, Lanzhou,

Gansu 730070, China

2 Gansu Research Center for Swine Production Engineering and Technology, Lanzhou, Gansu 730070, China

ORCID

Wei Wang

https://orcid.org/0000-0003-0960-8824 Pengfei Wang

https://orcid.org/0000-0002-9886-6982 Kaihui Xie

https://orcid.org/0000-0003-0463-2147

Ruirui Luo

https://orcid.org/0000-0003-1147-2911 Xiaoli Gao

https://orcid.org/0000-0003-4908-1051 Zunqiang Yan

https://orcid.org/0000-0001-8701-0647

Xiaoyu Huang

https://orcid.org/0000-0001-9467-3406

Qiaoli Yang

https://orcid.org/0000-0003-4577-6366

Shuangbao Gun

https://orcid.org/0000-0001-6242-7550

Submitted May 11, 2020; Revised Jul 30, 2020; Accepted Sept 2, 2020
Objective: microRNAs (miRNAs) can play a role in a variety of physiological and pathological processes, and their role is achieved by regulating the expression of target genes. Our previous high-throughput sequencing found that ssc-miR-185 plays an important regulatory role in piglet diarrhea, but its specific target genes and functions in intestinal porcine epithelial cell (IPEC-J2) are still unclear. We intended to verify the target relationship between porcine miR-185 and cell division cycle 42 (CDC42) gene in IPEC-J2 and to explore the effect of miR-185 on the proliferation of IPEC-J2 cells.

Methods: The TargetScan, miRDB, and miRanda software were used to predict the target genes of porcine miR-185, and CDC42 was selected as a candidate target gene. The CDC423 ' UTR-wild type (WT) and CDC42-3'UTR-mutant type (MUT) segments were successfully cloned into pmirGLO luciferase vector, and the luciferase activity was detected after co-transfection with miR-185 mimics and pmirGLO-CDC42-3'UTR. The expression level of CDC42 was analyzed using quantitative polymerase chain reaction and Western blot. The proliferation of IPEC-J2 was detected using cell counting kit-8 (CCK-8), methylthiazolyldiphenyltetrazolium bromide (MTT), and 5-ethynyl-2'-deoxyuridine (EdU) assays.

Results: Double enzyme digestion and sequencing confirmed that CDC42-3'UTR-WT and CDC42-3'UTR-MUT were successfully cloned into pmirGLO luciferase reporter vector, and the luciferase activity was significantly reduced after co-transfection with miR-185 mimics and CDC42-3'UTR-WT. Further we found that the mRNA and protein expression level of CDC42 were down-regulated after transfection with miR-185 mimics, while the opposite trend was observed after transfection with miR-185 inhibitor $(\mathrm{p}<0.01)$. In addition, the CCK-8, MTT, and EdU results demonstrated that miR-185 promotes IPEC-J2 cells proliferation by targeting CDC42.

Conclusion: These findings indicate that porcine miR-185 can directly target CDC42 and promote the proliferation of IPEC-J2 cells. However, the detailed regulatory mechanism of miR-185/CDC42 axis in piglets' resistance to diarrhea is yet to be elucidated in further investigation.

Keywords: ssc-miR-185; Cell Division Cycle 42 (CDC42); Target Relationship; Proliferation; Intestinal Porcine Epithelial Cell (IPEC-J2)

\section{INTRODUCTION}

Diarrhea is the main cause of death of newborn and suckling piglets, which brings enormous economic loss to the pig industry [1]. The occurrence of piglet diarrhea is related to a combination of genetic and improper management factors, especially the infection of pathogenic microorganisms, such as Escherichia coli [2], Salmonella [3], Clostridium perfringens [4-6], porcine epidemic diarrhea virus [7], etc. Therefore, it is necessary to search for molecular markers of diarrhea resistance and carry out porcine disease-resistant breeding. 
MicroRNAs (miRNAs), a class of small and endogenous noncoding RNA molecules with 19-25 nucleotides, can play a key role in post-transcription by binding to the 3 '-untranslated region (3'UTR) of target mRNA $[8,9]$. It can play roles in multiple biological processes, such as the cell proliferation [10], apoptosis [11], tumorigenesis [12], and immune inflammation [13] by suppressing the expression of its target genes.

In our previous study, we researched the expression profiles of the ileum miRNAs of 7 days piglets infected with Clostridium perfringens type C using small RNA-Seq, and found that ssc-miR-185 was differentially expressed between the resistant group and susceptible group of diarrhea piglets [14]. It is reported that miR-185 can play important roles in a variety of cancers, covering pancreatic cancer [15], bladder cancer [16], non-small cell lung cancer [17], prostate carcinoma [18], gastric cancer [19], breast cancer [20], hepatocellular carcinoma [21], and colorectal cancer [22]. In addition, miR185 can also play a regulatory role in response the immune inflammatory. Liu et al [23] analyzed microRNAs in alcoholic liver diseases using microarrays and found that miR185 can participate in immune response, inflammatory response and glutathione metabolism. Ma et al [24] found that the CCAT1/miR-185-3p/MLCK signaling pathway damages intestinal barrier function and promotes the deterioration of inflammatory bowel disease. Based on this, we speculate that miR-185 also plays an important role in the resistance of piglets to diarrhea infection.

Cell division cycle 42 (CDC42) is one of the members of Rho GTPase family [25]. It is reported that CDC42 regulates cell cytoskeleton and adhesion, cell functions, which are crucial in the development of various cancer diseases [26]. The research reported that miR-137 may directly target CDC42, inducing G1 cell cycle arrest and inhibiting the proliferation and invasion activities of colorectal cancer cells [27]. Moreover, miR-185 is a negative regulator of RhoA and CDC42, and could inhibit the proliferation and invasion of human colorectal cancer cells [28]. However, the function of miR$185 / C D C 42$ in intestinal porcine epithelial cell (IPEC-J2) remains to be determined.

In our current study, the relationship between porcine miR-185 and CDC42 was investigated in IPEC-J2. We predicted the target relationship between miR-185 and CDC42 using bioinformatics software. The mRNA and protein expression level of CDC42 in IPEC-J2 were detected after transfection with miR-185. The luciferase activity of recombinant plasmids was also detected. In addition, the effects of overexpression miR-185 or knockdown CDC42 on proliferation activity of IPEC-J2 were explored. In this study, our results show that porcine miR-185 can directly target CDC42 and promote the proliferation of IPEC-J2 cells.

\section{MATERIALS AND METHODS}

\section{Ethics statement}

All animal experiments were conducted according to the Regulations and Guidelines for Experimental Animals established by the Ministry of Science and Technology (Beijing, China, revised in 2004) and approved by the Committee for Animal Ethics of the College of Animal Science and Technology, Gansu Agricultural University (approval number 2006-398).

\section{Sample collection and cell culture}

The liver tissue samples were collected from three male landrace at six months and stored at $-80^{\circ} \mathrm{C}$ until RNA extraction and as a template for CDC42 gene 3'UTR amplification. The 293T cells and IPEC-J2 were purchased from BeNa Culture Collection (BNCC, Beijing, China). The cells were cultured in DMEM/F12 medium (HyClone, New York, NY, USA) supplemented with 10\% fetal bovine serum (Gibco, Thermo Fisher Scientific, Inc., New York, USA), and 1\% penicillin-streptomycin at $37^{\circ} \mathrm{C}$ and $5 \% \mathrm{CO}_{2}$. When cell confluence reached $70 \%$ to $80 \%$, the transfection is carried out.

\section{Total RNA extraction and cDNA synthesis}

Total RNA was extracted from porcine liver tissues and IPECJ2 cells using TransZol Up reagent (TransGen Biotech, Beijing, China) according to the manufacturer's instructions. Subsequently, the cDNA was synthesized by reverse transcription using PrimeScript RT reagent kit with gDNA Eraser (TaKaRa, Dalian, China) and stored at $-20^{\circ} \mathrm{C}$.

\section{Bioinformatic analysis}

Since the miR-185 is highly conserved among different species, the miRNA databases: TargetScan [29] (http://www. targetscan.org/vert_72/), miRDB [30] (http://www.mirdb. org/), and miRanda [31] (http://www.microrna.org/microrna/ home.do) online software were used to predict the target genes for porcine miR-185. Based on predictive criteria, being bound to targeted sequences with low free energy of binding and having good complementarity with targeted sequences, CDC42 was selected as a candidate mRNA for follow-up studies.

\section{Plasmid construction and dual-luciferase reporter assay}

To verify the targeting relationship between miR-185 and CDC42, a partial segment of the CDC42 mRNA 3'-UTR (WT) containing the miR-185 binding-sequence was polymerase chain reaction (PCR) amplified using specific primers (Table 1). A mutated segment of the CDC42 mRNA 3'-UTR (MUT) in which the miR-185 binding sequence TCTCTCC 
Table 1. Primers information in this study

\begin{tabular}{lllcc}
\hline Name & \multicolumn{1}{c}{ Accession no. } & \multicolumn{1}{c}{ Primer sequence (5'-3') } & Size (bp) & \multicolumn{1}{c}{ Region } \\
\hline CDC42-PCR (WT) & NM_001078680.1 & F: CCGCTCGAGTGTTGCACTGACTGGCTCTG & 188 & $1,373-1,392$ \\
& & R: ACGCGTCGACCGGGCAGTGGCATCCTTATT & $1,560-1,540$ \\
CDC42-qPCR & NM_001078680.1 & F: GACAGATTACGACCGCTGAGT & 151 & $193-213$ \\
& & R: TCCCAACGAGCAAGAAAGGAG & $343-323$ \\
B-actin-qPCR & XM_003124280.5 & F: ATATTGCTGCGCTCGTGGT & 148 & $142-160$ \\
& & R: TAGGAGTCCTTCTGGCCCAT & $289-270$ \\
\hline
\end{tabular}

The bold and underline indicate restriction enzyme sites.

CDC42, cell division cycle 42; PCR, polymerase chain reaction; WT, wild type; qPCR, quantitative PCR.

was converted to AGAGAGG was obtained using gene synthesis and subcloning (GENEWIZ, Suzhou, China). The PCR products were cloned into the pmirGLO (7,350 bp) dual luciferase reporter vector (Promega, Madison, WI, USA). The recombinant plasmids were confirmed by double enzyme digestion with Xho I and Sal I (TaKaRa, China) and sequencing.

For transfection, the $293 \mathrm{~T}$ cells reached $70 \%$ to $80 \%$ confluences, cells were incubated in 24 -well plates. The recombinant plasmids were co-transfected with miR-185 mimics (50 nM) and inhibitor (100 nM) using Lipofectamine 2000 reagent (Invitrogen, Carlsbad, CA, USA) according to the manufacturer's protocol, respectively. The miR-185 mimics and inhibitor were designed and synthesized by RiboBio Biotech Co., Ltd. (RiboBio, Guangzhou, China). After 48 h posttransfection, the luciferase activity was detected using the Dual Luciferase Reporter Assay System (Promega, USA). In this experiment, the pmirGLO vector was considered as a blank control, mimics NC and inhibitor NC were considered as a negative control. All reactions were performed in triplicate.

Quantitative polymerase chain reaction

The IPEC-J2 cells were collected after transfection with miR185 mimics and inhibitor. The quantitative polymerase chain reaction (qPCR) reaction was analyzed using TB Green Premix Ex Taq II (Tli RNaseH Plus) quantitative kit (TaKaRa, China) in Roche LightCycler 480 II instrument (Roche, Penzberg, Germany). The primer sequences are shown in Table 1. The thermal cycle for PCR was performed at $95^{\circ} \mathrm{C}$ for $30 \mathrm{sec}-$ onds, 40 cycles at $95^{\circ} \mathrm{C}$ for 5 seconds and $60^{\circ} \mathrm{C}$ for 30 seconds. The relative mRNA expression of $C D C 42$ gene was normalized with $\beta$-actin $(A C T B)$ gene, and the results were calculated using the $2^{-\Delta \Delta \mathrm{Ct}}$ method [32].

\section{Western blotting}

After cell transfection $48 \mathrm{~h}$, total proteins were collected from the treated cells by RIPA buffer (Solarbio, Beijing, China) and quantified using the BCA protein assay kit (Solarbio, Beijing, China). The each group of denatured proteins were loaded into $10 \%$ sodium dodecyl sulfate-polyacrylamide gelelectrophoresis, and transferred onto polyvinylidene fluoride membrane. Then, the membranes were blocked in Tris-buffered saline with Tween-20 and incubated with 5\% skim milk at room temperature for $1 \mathrm{~h}$. Next the membranes were incubated with primary antibodies (anti-CDC42, bs-3555R, 1:1,000; anti- $\beta$-actin, bsm-33036M, 1:1,500, Bioss, Beijing, China) at $4^{\circ} \mathrm{C}$ overnight. The membranes were then incubated with secondary antibodies (HRP, goat anti-rabbit IgG, bs-0295G-HRP, 1:2,000, Bioss, Beijing, China) for $2 \mathrm{~h}$ at room temperature. The final protein bands were visualized by enhanced chemiluminescence, and the gray level of the protein bands was analyzed using ImageJ software (National Institutes of Health, Bethesda, MD, USA).

\section{Interference RNA synthesis and overexpression vector construction}

The interference RNAs used in this experiment were designed and synthesized by GenePharma Company (Shanghai, China). The si-NC was regarded as a negative control. The interference sequences were shown in Table 2. The CDC42 gene was cloned into pcDNA3.1 (+) vector with Nhe I and $\mathrm{BmaH}$ I restriction sites. The pcDNA-CDC42 overexpression vector was constructed by GENEWIZ Company (Suzhou, China).

Table 2. The information of interference RNA sequence

\begin{tabular}{lll}
\hline Name & Sense $\mathbf{( 5}^{\prime} \mathbf{- 3}^{\prime} \mathbf{)}$ & Antisense $\mathbf{( 5}^{\prime} \mathbf{- 3}^{\prime} \mathbf{)}$ \\
\hline Si-CDC42-1 & CCUACACGACAAACAAAUUTT & AAUUUGUUUGUCGUGUAGGTT \\
Si-CDC42-2 & GCUCGUUGGGACCCAAAUUTT & AAUUUGGGUCCCAACGAGCTT \\
Si-NC & UUCUCCGAACGUGUCACGUTT & ACGUGACACGUUCGGAGAATT \\
\hline
\end{tabular}

CDC42, cell division cycle 42 


\section{Cell counting kit-8 assay}

Cell counting kit-8 (CCK-8, Beyotime, Shanghai, China) was used to detect cell viability. The $2 \times 10^{3}$ cells per well were inoculated in the 96-well plates and maintained in the incubator for $24 \mathrm{~h}$. Subsequently, the cells were treated with miR-185 mimics, mimics NC, miR-185 inhibitor and inhibitor NC for $24 \mathrm{~h}$, respectively. Add $10 \mu \mathrm{L}$ of CCK- 8 reagent to each well according to the manufacturer's instructions and incubated for another $4 \mathrm{~h}$. Finally, cell viability was determined by measuring the optical density (OD) at $450 \mathrm{~nm}$.

\section{Methylthiazolyldiphenyl-tetrazolium bromide assay}

The methylthiazolyldiphenyl-tetrazolium bromide (MTT, Beyotime, Shanghai, China) was also used to examine cell viability. The $5 \times 10^{3}$ cells per well were cultured for $24 \mathrm{~h}$ in 96-well plates before treatment with miR-185 mimics, mimics NC, miR-185 inhibitor and inhibitor NC. Then they were incubated for $24 \mathrm{~h}$ at $37^{\circ} \mathrm{C}$ containing $5 \% \mathrm{CO}_{2}$. Followed by $10 \mu \mathrm{L}$ of MTT reagent $(5 \mathrm{mg} / \mathrm{mL})$ added to per well for another $4 \mathrm{~h}$. The medium was discarded after $4 \mathrm{~h}$ of treatment and the formazan crystals were dissolved using $110 \mu \mathrm{L}$ of dimethyl sulfoxide. The wavelength at $490 \mathrm{~nm}$ was selected, and the OD was determined using SkanIt microplate reader (Thermo Fisher Scientific Inc., USA).

\section{5-ethynyl-2'-deoxyuridine assay}

The BeyoClick EdU Cell Proliferation Kit with Alexa Fluor 555 (EdU, Beyotime, Shanghai, China) was used to detect cell proliferation. After seeding in 24 -well plates $\left(5 \times 10^{3}\right.$ cells per well) for $24 \mathrm{~h}$, the IPEC-J2 cells were transfected. After transfected $24 \mathrm{~h}$, the cells were incubated with $10 \mu \mathrm{M}$ EdU solution in growth medium for $2 \mathrm{~h}$. Then, the cells stained with Azide 555 solution (red) and Hoechst 33342 (blue). Finally, the results were observed under a fluorescence microscope (Olympus IX71, Tokyo, Japan) with $200 \times$ magnification. The EdU positive cells were analyzed with the ImageJ software.

\section{Statistical analysis}

The IBM SPSS Statistics software (version 21.0; IBM, Armonk, NY, USA) was used to analyze the data, all experiments were repeated at least three times. A Student's t-test was applied to compare two groups and one-way analysis of variance (ANOVA) was performed for multiple groups. All values in this study were expressed as the mean \pm standard deviation, a $\mathrm{p}$ value of less than 0.05 was indicated statistical significance.

\section{RESULTS}

\section{Predicting targeted mRNA}

To explore the potential mechanism of ssc-miR-185, we performed a multi-sequence alignment analysis of the mature
miR-185 sequences in different species and found that the mature sequences of miR-185 was highly conserved in vertebrates (Figure 1A). The targeting mRNAs of miR-185 were predicted using TargetScan, miRDB and miRanda software, and 385, 1,137, and 1,225 target genes were obtained respectively, and 100 common target genes were obtained by the intersection (Figure 1B). It was found that $C D C 42$ gene 3 'UTR can complement and bind to the seed region of miR-185 (Figure 1C). The CDC42 gene 3'UTR partial sequences contain miR-185 binding sites as showed in Figure 1D.

\section{Recombinant plasmids identification and luciferase activity detection}

Double enzyme digestion and sequencing confirmed that CDC42-3'UTR-WT and CDC42-3'UTR-MUT were successfully cloned into pmirGLO luciferase reporter vector (Figure 2A-2D). The TCTCTCC sequences were successfully mutated to AGAGAGG, without changes to other bases. In order to confirm the role of miR-185 in regulating CDC423'UTR, the luciferase activity was detected using the Dual Luciferase Reporter Assay System according to specification. We found that miR-185 mimics remarkably reduced the luciferase activity of the pmirGLO-CDC42-WT $(\mathrm{p}<0.01)$, but not that of the pmirGLO and pmirGLO-CDC42-MUT ( $\mathrm{p}>$ 0.05) (Figure 3).

\section{Effects of miR-185 on CDC42 expression level in IPEC-J2}

To further confirm the effects of miR-185 on CDC42, qPCR and Western blot analyses were used to examine the mRNA and protein expression in IPEC-J2 cells after transfected with miR-185 mimics, mimics NC, miR-185 inhibitor and inhibitor NC, respectively. The results showed that the mRNA and protein expression level of CDC42 was dramatically decreased when transfected with miR-185 mimics than in transfected with mimics NC $(\mathrm{p}<0.01)$, however, the CDC42 expression level both mRNA and protein were significantly increased when transfected with miR-185 inhibitor than in transfected with inhibitor NC $(\mathrm{p}<0.01)$ (Figure 4A-4C). These results demonstrate that miR-185 directly regulates CDC42 expression.

\section{miR-185 promotes the IPEC-J2 cell proliferation}

In order to explore the effect of miR-185 on IPEC-J2 cell proliferation, CCK-8 assay and MTT assay were used to detect the cell viability after transfected with miR-185 mimics, mimics NC, miR-185 inhibitor and inhibitor NC. We found that overexpression miR-185 enhanced cell viability, while knockdown miR-185 can inhibit cell viability of IPEC-J2 cells. The CCK-8 and MTT assays showed similar expression trends (Figure 5A, 5B). The EdU assay was used to detected cell proliferation, and the results showed that the EdU positive 
A

Species name
ssc-miR-185
hsa-miR-185-5p
mmu-miR-185-5p
mo-miR-185-5p
mml-miR-185-5p
ggo-miR-185
bta-miR-185
ocu-miR-185-5p

B

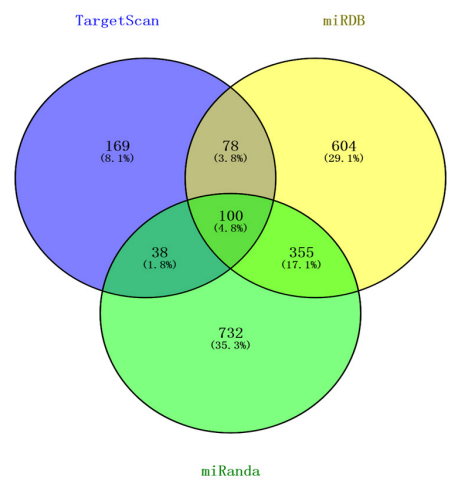

Accession

Mature sequence

MIMAT0007759

UGGAGAGAAAGGCAGUVCCUGA

MIMAT0000455 UGGAGAGA A AGCAGUUCCUGA

MIMAT0000214

MIMAT0000862

MIMAT0006219

MIMAT0024086

MIMAT0009247

MIMAT0048291

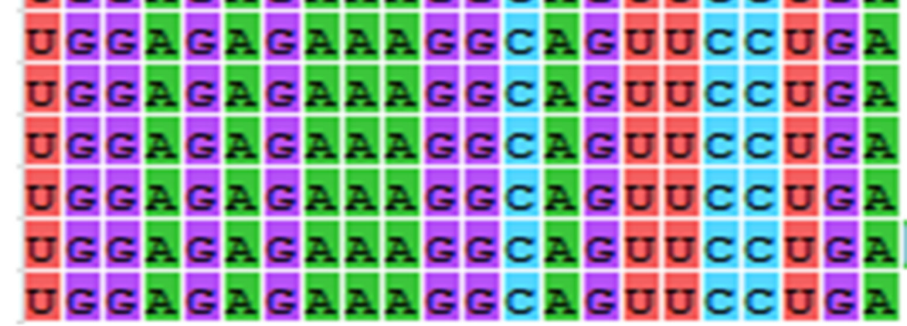

C

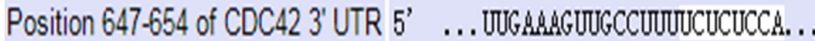 \\ hsa-miR-185-5p \\ $3^{\prime}$ \\ AGUCCUUGACGGA-AGAGAGU \\ ||||||||||||
}

D

>NM_001078680.1 CDC42-3'UTR

TGTTGCACTGACTGGCTCTGGTTTTATGTATGTATCAG

Primer-F

GTGCCCTCATTTCTGCTTCCTTGAATCCCCTTGGCTCTGT

CTAATTGGTGGGATAAAAGGAGTTCAGAGTGAATATTTT

TCATGCAGCCACTGTAATTGCTCTCTCCATCTTTGCCTG

Binding site

CGCCTTCTGTATAATAAGGATGCCACTGCCCG

Primer-R

Figure 1. Bioinformatics analysis. (A) miR-185 mature sequences alignment in different species. (B) Venn diagram of target gene intersection. (C) Predicting the binding sites between miR-185 and CDC42 using TargetScan software. (D) CDC42 gene 3'UTR partial sequences. The black underline indicates the amplification primer and the red underline indicates the binding sites. CDC42, cell division cycle 42; UTR, untranslated region.

cells were significantly increased after transfected with miR185 mimics. On the contrary, after transfected with miR-185 inhibitor, the EdU positive cells were significantly reduced. Therefore, we conclude that miR-185 can promote IPEC-J2 cells proliferation.

Knockdown CDC42 promotes the proliferation of IPEC-J2 cells

To confirm whether miR-185 directly promotes proliferation of IPEC-J2 cells by targeting CDC42, we compared knockdown and overexpression of CDC42 in IPEC-J2 cells. After transfected with si-CDC42-1 and si-CDC42-2, the expression of CDC42 was downregulated by 0.828 and 0.238 fold. So, the si-CDC42-2 was used in subsequent experiments. However, after transfected with pcDNA-CDC42 plasmid, the expression of CDC42 was significantly upregulated (Figure 6A). The CCK-8, MTT, and EdU assays were used to detect cell vitality and cell proliferation, these results showed that 
A

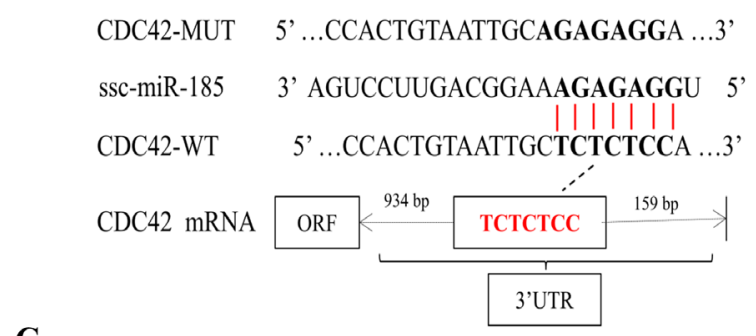

C

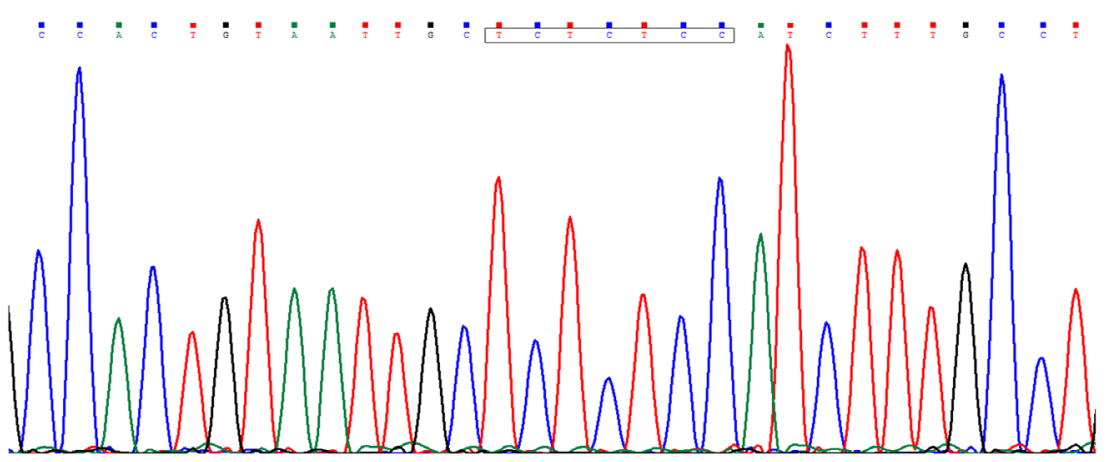

D

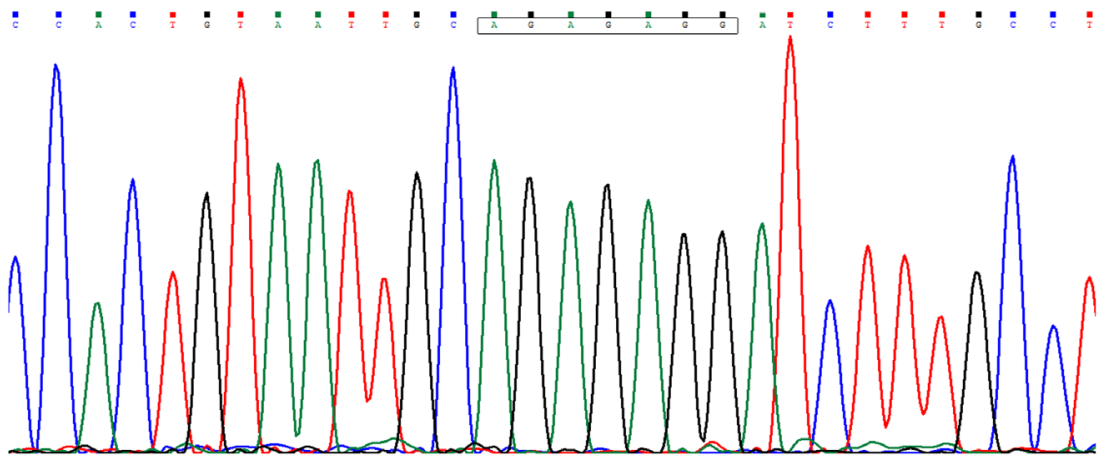

Figure 2. Construction and identification of recombinant plasmids. (A) The location information between miR-185 and CDC42 wild-type and mutant-type. (B) Recombinant plasmid enzyme digestion identification. M1, 5 kb DNA marker; 1 and 2, wild-type and mutant-type recombinant plasmids; M2, 10 kb DNA marker. (C) CDC42-3'UTR-wild type sequencing. The box represents the binding sites. (D) CDC42-3'UTR-mutant type sequencing. The box represents the mutant sites. CDC42, cell division cycle 42; UTR, untranslated region.

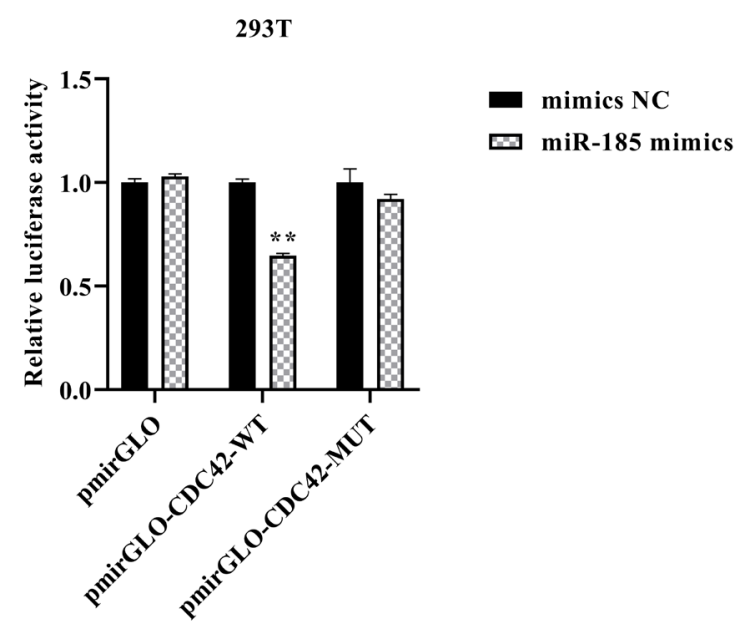

Figure 3. The relative luciferase activity of CDC42-3'UTR. ** Means $p<0.01$. CDC42, cell division cycle 42; UTR, untranslated region. knockdown CDC42 promoted cell proliferation, while overexpression CDC42 inhibited proliferation of IPEC-J2 cells (Figure 6B, 6C, 6D, and 6E). Therefore, we hypothesized that miR-185 might directly target CDC42 to promote IPEC-J2 cell proliferation.

\section{DISCUSSION}

Diarrhea is a common disease in pig industry, especially harmful to piglets. Our previous study found that ssc-miR-185 was up-regulated in the resistance group of diarrhea piglets [14]. We speculated that it may play an important role in resisting diarrhea, but the specific target gene is unknown. It is well known that bioinformatics prediction combined with experimental validation is an effective method for screening 
A

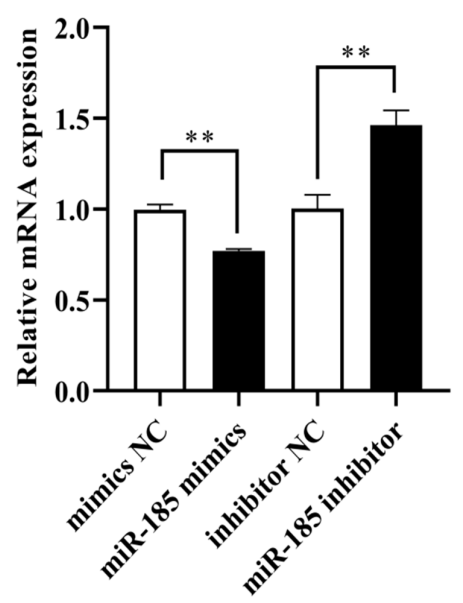

B

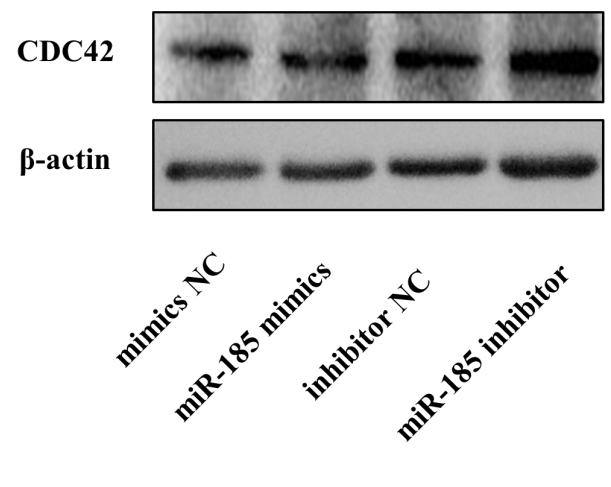

C

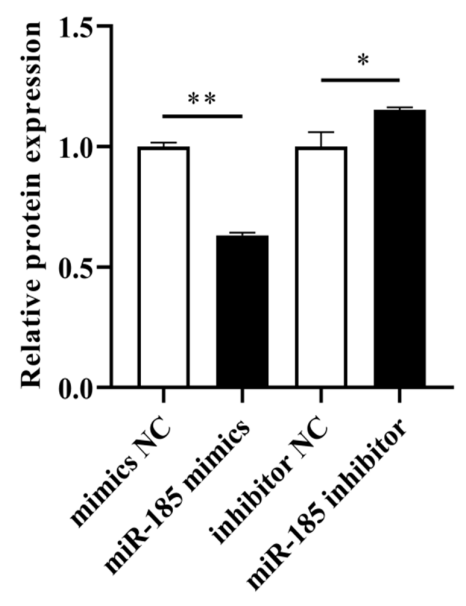

Figure 4. The relative expression level of CDC42. (A) mRNA expression level of CDC42. (B and C) protein expression level of CDC42. CDC42, cell division cycle 42. All ** means $p<0.01$, * means $p<0.05$, $\beta$-actin was detected as an internal control, $n=3$.

A

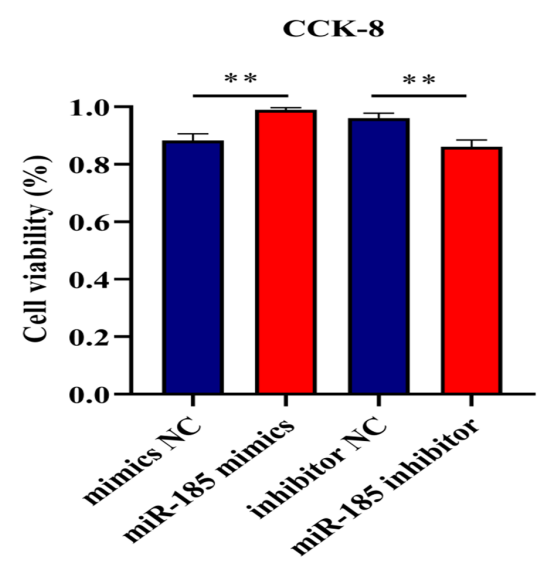

C

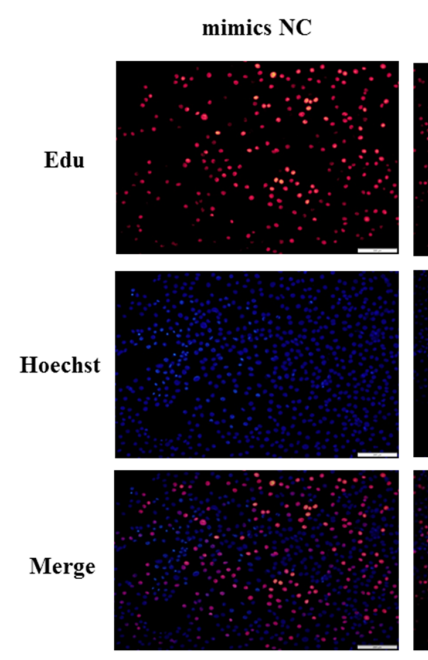

miR-185 mimics
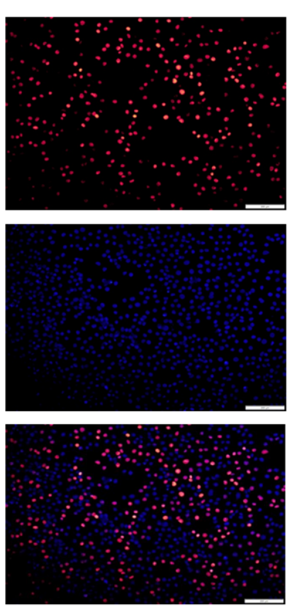

B

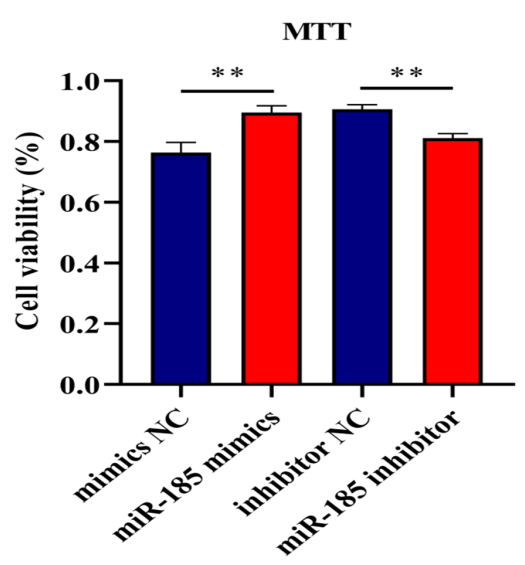

D

Figure 5. The effect of miR-185 on IPEC-J2 cells proliferation. (A) The results of CCK-8 assay. (B) The results of MTT assay. (C) The results of EdU assay. (D) The count of EdU positive cells. CCK-8, cell counting kit-8; MTT, methylthiazolyldiphenyl-tetrazolium bromide. All $\star \star ~ i n d i c a t e s ~ p<0.01$. 
A

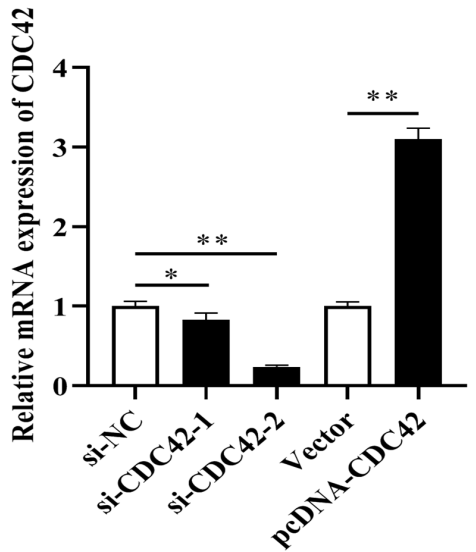

D

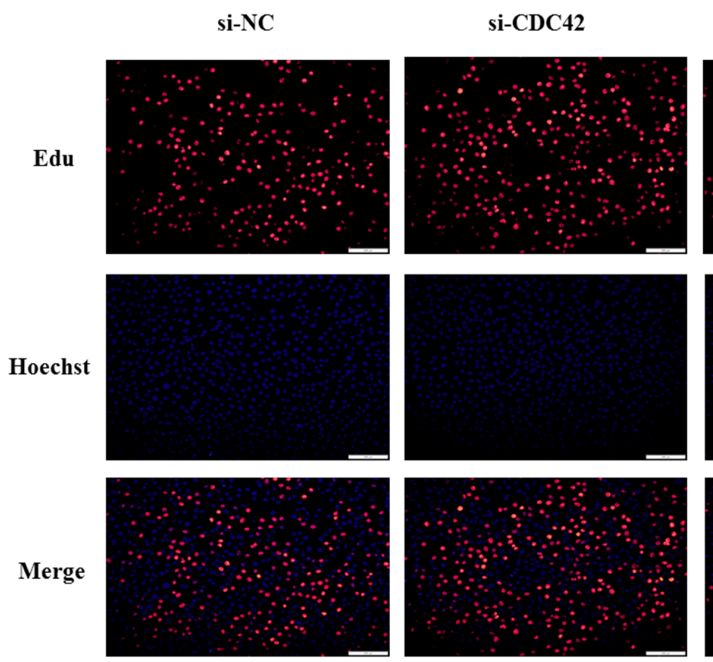

B

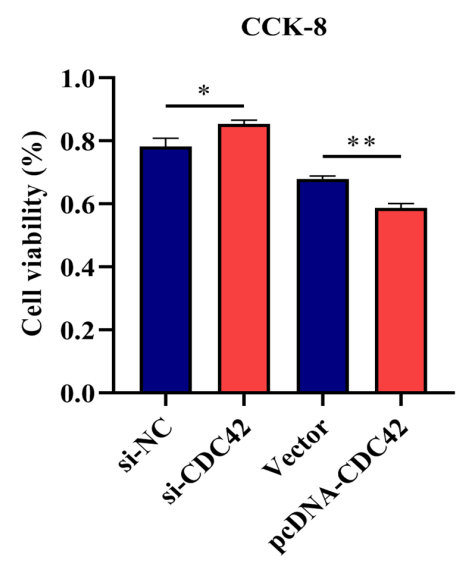

C

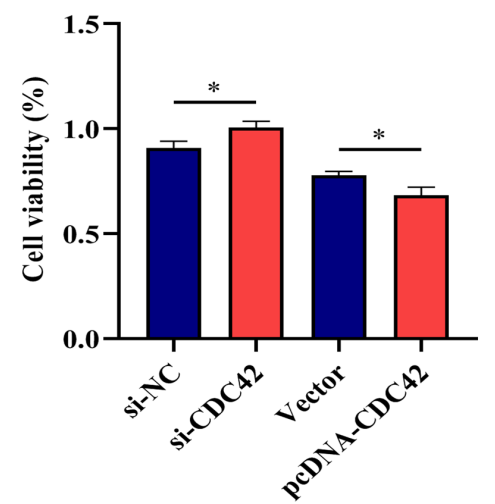

$\mathbf{E}$

pcDNA3.1 vector
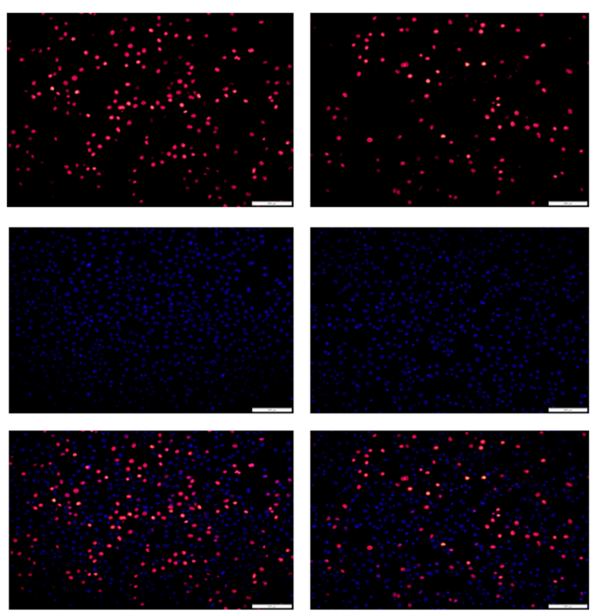

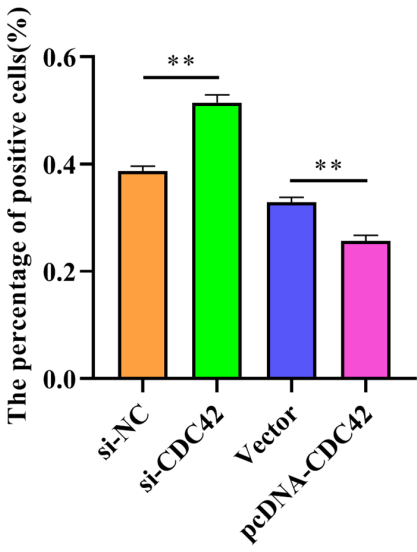

Figure 6. The effect of CDC42 on IPEC-J2 cells proliferation. (A) The interference and overexpression efficiency of CDC42 gene. (B) The results of CCK-8 assay. (C) The results of MTT assay. (D) The results of EdU assay. (E) The count of EdU positive cells. CDC42, cell division cycle 42; CCK-8, cell counting kit-8; MTT, methylthiazolyldiphenyl-tetrazolium bromide. All ** means $p<0.01$, * means $p<0.05$.

miRNA target genes. In this study, three softwares: TargetScan, miRDB, and miRanda were used to predict the target genes for miR-185, which could effectively reduce the false positive rate. By finding the intersection, CDC42 was selected as a candidate target gene.

Previous research has shown that CDC42 is a potential target of miR-185. For example, Zhang et al [21] confirmed that CDC42 is a direct target of miR-185 in human hepatocellular carcinoma using luciferase reporter assays. Liu et al [28] showed that miR-185 expression significantly suppressed the RhoA and CDC42 3'UTR activities using a luciferase-reporter assay, and could inhibit the proliferation and invasion of human colorectal cancer cells. Notably, the miR-185 and CDC42 gene sequence are highly conserved between pig and human. Hence, we assumed that ssc-miR-185 could be binding to the conserved sites of CDC42. In our present study, we found that CDC42-3'UTR contained miR-185 binding site according to the bioinformatics software. The luciferase activity is remarkably suppressed in pmirGLO-CDC42-WT group after transfection with miR-185 mimics. These results indicated that CDC42 was a target gene of porcine miR-185. As a chemokine that mediates tumors, CDC42 can participate in the migration and invasion of various cancer cells [33]. Previous research reported that microRNA-384 inhibits proliferation, migration and invasion of glioma by targeting at CDC42 [34]. Yang et al [35] confirmed that downregulation of miR-25 markedly inhibited A549 cell proliferation, induced G1 cell cycle arrest, by targeting CDC42. In addition, miR-330 regulates the proliferation of colorectal cancer cells by targeting CDC42 [36].

More and more studies have confirmed that miRNA can negatively regulate the expression of target genes. Niu et al 
[37] demonstrated that ROCK2 was negatively associated with miR-185-5p and promoted hepatocellular carcinoma cell migration and invasion. Fang et al [38] revealed that the expression level of miR-185 and STIM1 were negatively correlated as detected by qRT-PCR and Western blot assays. In this study, the mRNA and protein expression level of CDC42 were dramatically decreased after overexpression of miR-185, which further confirms the targeting relationship between the porcine miR-185 and CDC42. Functionally, miR-185 has been reported to inhibit the proliferation of cancer cells and promote apoptosis. For example, upregulation of miR185 promotes apoptosis of the human gastric cancer cell line MGC803 [39]. Zou et al [40] found that RKIP through up-regulation of miR-185 suppresses the proliferation and metastasis of breast cancer cell lines. Furthermore, miR-185 can inhibit virus infection through the regulation of immunometabolic pathways [41]. In our present research, we detected the exact function of miR-185 for proliferation and proved that miR- 185 promoted the proliferation of normal IPEC-J2 cells. However, whether miR-185 can resist piglet diarrhea caused by pathogenic bacteria infection and inhibit intestinal cell apoptosis requires further research. In summary, our results may provide new insights into the screening of miR-185/CDC42 molecular markers.

\section{CONCLUSION}

In conclusion, luciferase activity, qPCR and Western blot assays displayed that porcine miR-185 can directly target CDC42 gene. In addition, overexpression miR-185 and knockdown CDC42 can promote cell proliferation of IPEC-J2. However, the detailed regulatory mechanism of miR-185/ CDC42 axis in piglets' resistance to diarrhea requires further investigation.

\section{CONFLICT OF INTEREST}

We certify that there is no conflict of interest with any financial organization regarding the material discussed in the manuscript.

\section{ACKNOWLEDGMENTS}

This research was funded by the Discipline Construction Fund Project of Gansu Agricultural University (GSAUXKJS-2018-042).

\section{REFERENCES}

1. Huang X, Yang Q, Yuan J, et al. Effect of genetic diversity in swine leukocyte antigen-DRA gene on piglet diarrhea. Genes 2016;7:36. https://doi.org/10.3390/genes7070036
2. Dai C, Yang L, Jin J, Wang H, Wu S, Bao W. Regulation and molecular mechanism of TLR5 on resistance to Escherichia coli F18 in weaned piglets. Animals 2019;9:735. https://doi. org/10.3390/ani9100735

3. Xia B, Yu J, He T, et al. Lactobacillus johnsonii L531 ameliorates enteritis via elimination of damaged mitochondria and suppression of SQSTM1-dependent mitophagy in a Salmonella infantis model of piglet diarrhea. FASEB J 2020;34:2821-39. https://doi.org/10.1096/f.201901445RRR

4. Huang XY, Sun WY, Yan ZQ, et al. Novel insights reveal antimicrobial gene regulation of piglet intestine immune in response to Clostridium perfringens infection. Sci Rep 2019;9: 1963. https://doi.org/10.1038/s41598-018-37898-5

5. Huang X, Sun W, Yan Z, et al. Integrative analyses of long non-coding RNA and mRNA involved in piglet ileum immune response to Clostridium perfringens type $\mathrm{C}$ infection. Front Cell Infect Microbiol 2019;9:130. https://doi.org/10. 3389/fcimb.2019.00130

6. Yan Z, Cai L, Huang X, et al. Histological and comparative transcriptome analyses provide insights into small intestine health in diarrheal piglets after infection with Clostridium perfringens type C. Animals 2019;9:269. https://doi.org/10. 3390/ani9050269

7. Wu M, Zhang Q, Yi D, et al. Quantitative proteomic analysis reveals antiviral and anti-inflammatory effects of puerarin in piglets infected with porcine epidemic diarrhea virus. Front Immunol 2020;11:169. https://doi.org/10.3389/fimmu.2020. 00169

8. Ambros V. The functions of animal microRNAs. Nature 2004; 431:350-5. https://doi.org/10.1038/nature02871

9. Bartel DP. MicroRNAs: genomics, biogenesis, mechanism, and function. Cell 2004;116:281-97. https://doi.org/10.1016/ S0092-8674(04)00045-5

10. Chang W, Zhang L, Xian Y, Yu Z. MicroRNA-33a promotes cell proliferation and inhibits apoptosis by targeting PPARa in human hepatocellular carcinoma. Exp Ther Med 2017;13: 2507-14. https://doi.org/10.3892/etm.2017.4236

11. Liu Y, Liang H, Jiang X. miR-1297 promotes apoptosis and inhibits the proliferation and invasion of hepatocellular carcinoma cells by targeting HMGA2. Int J Mol Med 2015;36: 1345-52. https://doi.org/10.3892/ijmm.2015.2341

12.Zhu T, Fan D, Ye K, et al. Role of miRNA-542-5p in the tumorigenesis of osteosarcoma. FEBS Open Bio 2020;10:627-36. https://doi.org/10.1002/2211-5463.12824

13. Chandan K, Gupta M, Sarwat M. Role of host and pathogenderived micrornas in immune regulation during infectious and inflammatory diseases. Front Immunol 2020;10:3081. https://doi.org/10.3389/fimmu.2019.03081

14. Wang P, Huang X, Yan Z, et al. Analyses of miRNA in the ileum of diarrheic piglets caused by Clostridium perfringens type C. Microb Pathog 2019;136:103699. https://doi.org/10. 1016/j.micpath.2019.103699 
15. Wang YP, Huang Y, Hou T, Lu M. LncRNA XIST acts as a ceRNA sponging miR-185-5p to modulate pancreatic cancer cell proliferation via targeting CCND2. Transl Cancer Res 2020;9:1427-38. https://doi.org/10.21037/tcr.2020.01.26

16.Lin G, Zhang C, Chen X, et al. Identification of circulating miRNAs as novel prognostic biomarkers for bladder cancer. Math Biosci Eng 2019;17:834-44. https://doi.org/10.3934/ mbe.2020044

17. Ge P, Cao L, Yao YJ, Jing RJ, Wang W, Li HJ. IncRNA FOXD2AS1 confers cisplatin resistance of non-small-cell lung cancer via regulation of miR185-5p-SIX1 axis. Onco Targets Ther 2019;12:6105-17. https://doi.org/10.2147/OTT.S197454

18. Ostadrahimi S, Valugerdi MA, Hassan M, et al. miR-1266-5p and miR-185-5p promote cell apoptosis in human prostate cancer cell lines. Asian Pac J Cancer Prev 2018;19:2305-11. https://doi.org/10.22034/APJCP.2018.19.8.2305

19. Tan B, Li Y, Zhao Q, Fan L, Wang D. ZNF139 increases multidrug resistance in gastric cancer cells by inhibiting miR-185. Biosci Rep 2018;38:BSR20181023. https://doi.org/10.1042/ BSR20181023

20.Liu L, Zhu Y, Liu AM, Feng Y, Chen Y. Long noncoding RNA LINC00511 involves in breast cancer recurrence and radioresistance by regulating STXBP4 expression via miR-185. Eur Rev Med Pharmacol Sci 2019;23:7457-68. https://doi. org/10.26355/eurrev_201909_18855

21.Zhang Q, Chen Y, Liu K. miR-185 inhibits cell migration and invasion of hepatocellular carcinoma through CDC42. Oncol Lett 2018;16:3101-7. https://doi.org/10.3892/ol.2018.8971

22. Yuan M, Zhang X, Zhang J, et al. DC-SIGN-LEF1/TCF1miR-185 feedback loop promotes colorectal cancer invasion and metastasis. Cell Death Differ 2020;27:379-95. https://doi. org/10.1038/s41418-019-0361-2

23. Liu Y, Chen SH, Jin X, Li YM. Analysis of differentially expressed genes and microRNAs in alcoholic liver disease. Int J Mol Med 2013;31:547-54. https://doi.org/10.3892/ijmm. 2013.1243

24. Ma D, Cao Y, Wang Z, et al. CCAT1 lncRNA promotes inflammatory bowel disease malignancy by destroying intestinal barrier via downregulating miR-185-3p. Inflamm Bowel Dis 2019;25:862-74. https://doi.org/10.1093/ibd/izy381

25.Etienne-Manneville S, Hall A. Rho GTPases in cell biology. Nature 2002;420:629-35. https://doi.org/10.1038/nature 01148

26.Sahai E, Marshall CJ. RHO-GTPases and cancer. Nat Rev Cancer 2002;2:133-42. https://doi.org/10.1038/nrc725

27. Liu M, Lang N, Qiu M, et al. miR-137 targets Cdc42 expression, induces cell cycle G1 arrest and inhibits invasion in colorectal cancer cells. Int J Cancer 2011;128:1269-79. https://doi. org/10.1002/ijc.25452

28.Liu M, Lang N, Chen X, et al. miR-185 targets RhoA and Cdc42 expression and inhibits the proliferation potential of human colorectal cells. Cancer Lett 2011;301:151-60. https:// doi.org/10.1016/j.canlet.2010.11.009

29. Lewis BP, Shih I, Jones-Rhoades MW, Bartel DP, Burge CB. Prediction of mammalian microRNA targets. Cell 2003;115: 787-98. https://doi.org/10.1016/S0092-8674(03)01018-3

30. Chen Y, Wang X. miRDB: an online database for prediction of functional microRNA targets. Nucleic Acids Res 2020;48: D127-31. https://doi.org/10.1093/nar/gkz757

31. Betel D, Wilson M, Gabow A, Marks DS, Sander C. The microRNA.org resource: targets and expression. Nucleic Acids Res 2008;36(Suppl 1):D149-53. https://doi.org/10. 1093/nar/gkm995

32.Livak KJ, Schmittgen TD. Analysis of relative gene expression data using real-time quantitative PCR and the $2^{-\triangle \Delta C T}$ method. Methods 2001;25:402-8. https://doi.org/10.1006/meth.2001. 1262

33. Chernichenko N, Omelchenko T, Deborde S, et al. Cdc42 mediates cancer cell chemotaxis in perineural invasion. Mol Cancer Res 2020;18:913-25. https://doi.org/10.1158/15417786.MCR-19-0726

34. Gu G, Wang L, Zhang J, Wang H, Tan T, Zhang G. MicroRNA384 inhibits proliferation migration and invasion of glioma by targeting at CDC42. Onco Targets Ther 2018;11:4075-85. https://doi.org/10.2147/OTT.S166747

35. Yang T, Chen T, Li Y, et al. Downregulation of miR-25 modulates non-small cell lung cancer cells by targeting CDC42. Tumor Biol 2015;36:1903-11. https://doi.org/10.1007/s13277014-2793-0

36.Li Y, Zhu X, Xu W, Wang D, Yan J. miR-330 regulates the proliferation of colorectal cancer cells by targeting Cdc42. Biochem Biophys Res Commun 2013;431:560-5. https:// doi.org/10.1016/j.bbrc.2013.01.016

37.Niu Y, Tang G. miR-185-5p targets ROCK2 and inhibits cell migration and invasion of hepatocellular carcinoma. Oncol Lett 2019;17:5087-93. https://doi.org/10.3892/ol.2019.10144

38. Fang M, Li Y, Wu Y, Ning Z, Wang X, Li X. miR-185 silencing promotes the progression of atherosclerosis via targeting stromal interaction molecule 1. Cell Cycle 2019;18:682-95. https://doi.org/10.1080/15384101.2019.1580493

39. Fan L, Tan B, Li Y, et al. Upregulation of miR-185 promotes apoptosis of the human gastric cancer cell line MGC803. Mol Med Rep 2018;17:3115-22. https://doi.org/10.3892/mmr.2017. 8206

40.Zou Q, Wu H, Fu F, Yi W, Pei L, Zhou M. RKIP suppresses the proliferation and metastasis of breast cancer cell lines through up-regulation of miR-185 targeting HMGA2. Arch Biochem Biophys 2016;610:25-32. https://doi.org/10.1016/ j.abb.2016.09.007

41.Singaravelu R, O'Hara S, Jones DM, et al. MicroRNAs regulate the immunometabolic response to viral infection in the liver. Nat Chem Biol 2015;11:988-93. https://doi.org/10.1038/ nchembio. 1940 